\title{
1 Genetical engineered lung cancer cell for analyzing 2 Epithelial-Mesenchymal transition
}

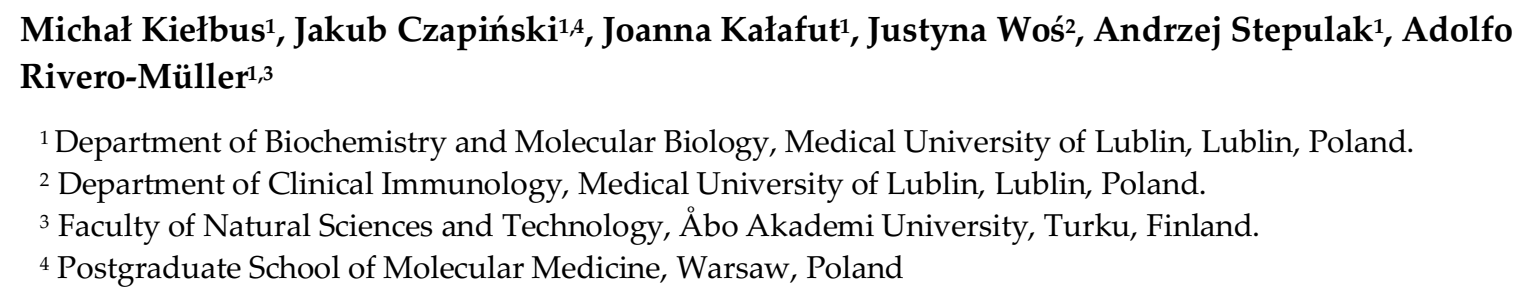

Abstract: Cell plasticity, defined as the ability to undergo phenotypical transformation in a reversible manner, is a physiological processes that also exert important roles in disease progression Two forms of cellular plasticity are epithelial-mesenchymal transition (EMT) and its inverse process, mesenchymal-epithelial transition (MET). These processes have been correlated to the poor outcome of different types of neoplasias as well as drug resistance development. Since EMT/MET are transitional processes, we have generated and validated a reporter cell line. Specifically, a far-red fluorescent protein was knocked-in in-frame with the mesenchymal gene marker VIMENTIN (VIM) in H2170 lung cancer cells. The vimentin reporter cells (VRCs) are a reliable model for studying EMT and MET showing cellular plasticity upon a series of stimulations. These cells are a robust platform to dissect the molecular mechanisms of these processes, and for drug discovery in vitro and in the future in vivo.

Keywords: epithelial-mesenchymal transition (EMT); mesenchymal-epithelial transition (MET); cancer cell line; vimentin; reporter;

\section{Introduction}

The ability of cells to temporally acquire different characteristics, also known as cell plasticity, plays essential roles in physiological and pathophysiological processes [1]. Epithelial cells might transform to a mesenchymal phenotype and return to epithelial phenotype by epithelial-mesenchymal transition (EMT) and mesenchymal-epithelial transition (MET), respectively. EMT has been correlated to the metastasis and invasion potential of many types of malignancies [2]. The fact that the poor outcome of many types of neoplasias correlates with EMT/MET, makes these molecular phenomena important focus for research and drug targeting [3-6]. Despite the clinical association, the role of EMT/MET in metastasis is inconclusive, for example mesenchymal-like prostate cancer cells survive in circulation but, unlike epithelial or cells undergoing EMT, they are unable to form macrometastases [7]. In fact, breast cancer cell metastasis to lung tissue in mice was not affected by decreasing EMT - by targeting EMT-triggering transcription factors such as SNAI1 (Snail) and SNAI2 (Slug) by overexpressing miR-200 [8]. An additional role of EMT in tumorigenesis might be the development of apoptotic tolerance and increased resistance to chemotherapy as has been found in animal models and patients [8,9]. Recently, a hybrid stage between epithelial and mesenchymal 
phenotypes (hybE/M) has been recognized, and such hybE/M cells migrate outside the primary tumors displaying some mesenchymal features such as spindle-like morphology, increased nuclear levels of ZEB1 transcription factor, and epithelial ones such as cell-cell adhesion potential [10,11].

In order to study the transitory stages of EMT, MET and hybE/M, in particular at in vivo settings, there is need to generate reporter cells to visualize phenotypical changes. There have been different approaches to this, for example the use of heterologous fluorescent proteins driven under stagespecific promoters such as mesenchymal (ZEB1) and epithelial (CDH1) [12], mesenchymal snai1 and epithelial sox10 in zebrafish [13], or mesenchymal Vimentin (Vim) in mice [14]. Nevertheless, heterologous expression of exogenous reporter genes is hampered by the existence of alternative promoters, cis- and trans-regulatory elements, and epigenetic events that modulates promoter activation making some data unreliable.

To overcome these limitations, we genetically engineered and characterized human lung carcinoma H2170 Vimentin Reporter Cells (VRCs) where the fluorescent protein coding gene (mCardinal) has been knocked-in as a genetic fusion, although separate proteins due to a T2A self-cleaving peptide, at both alleles of the mesenchymal marker VIM.

\section{Materials and Methods}

\subsection{Cell culture, nucleofection and transfection}

Human squamous lung carcinoma (H2170), human colorectal adenocarcinoma (HT29) as well as human embryonic kidney (HEK293) cells were cultured according to standard mammalian tissue culture protocols and sterile techniques. The cell lines were cultured in DMEM supplemented with $10 \%$ fetal calf serum (FCS), 100 units $/ \mathrm{mL}$ and penicillin $/ 100 \mu \mathrm{g} / \mathrm{mL}$ streptomycin. All tissue culture media and supplements were obtained from Gibco. H2170, HT29 and HEK293 cells were obtained from ATCC. H2170 cells were nucleofected for genome editing with the use of Nucleofector I Device and Cell Line Nucleofector Kit T (Amaxa). The optimized protocol is as follows: nulceofection of 1 million suspended cell with $2 \mu \mathrm{g}$ of the plasmid DNA using program X-001, generating transfection efficiency of $99 \%$. For performing functional experiments on a smaller scale the $\mathrm{H} 2170$ cells were transfected with the use of Lipofectamine 3000 (Invitrogen) with transfection efficiency $90-95 \%$ after $24 \mathrm{~h}$. 50,000 cells were seeded a day before transfection in 24-well plates. Transfection was performed using 500ng of the plasmid DNA, $1.5 \mu \mathrm{L}$ Lipofectamine ${ }^{\circledR 3} 3000$ Reagent and $1 \mu \mathrm{L}$ P3000 ${ }^{\mathrm{TM}}$ Reagent (both from Invitrogen) per well, following the manufacture's protocol. HEK293 were transfected with the use of TurboFect reagent (Thermo) according to the protocol supplied by the company. Next day, the transfection efficiency was in range $80-90 \%$.

\subsection{Plasmid vectors for the knock-in to VIM}

We modified Cas9/gRNA expressing plasmid pSpCas9(BB)-2A-Puro (PX459) V2.0 (Addgene plasmid \#62988) by inserting the fragment coding the targeting gRNA using digestion/ligation protocol [15]. The oligonucleotides used to generate the gRNA were gRNA-F and gRNA-R (Table S1). The template plasmid used for inserting DYKDDDDK-tagged (FLAG) mCardinal fluorescent protein gene after VIM was designed as following (gRNAsite-800nt of Vim-P2A-mCardinal-FLAG-800nt of 3'VimUTRgRNAsite) and was synthetized by Thermo. The Cas9-gRNA and the template plasmids were both nucleofected to cells at the same time and the cells were selected 2 days upon nucleofection for 
another 2 days in $5 \mu \mathrm{g} / \mathrm{ml}$ of puromycin. The positive single cell clones obtained by dilution were genotyped what was described in details in Genotyping section.

\subsection{Plasmid vectors used in functional assays}

For functional experiments in H2170 knocked-in cells we used (FLAG) Snail 6SA (active Snail) plasmid which was a gift from Mien-Chie Hung (Addgene plasmid \# 16221) [16], TGFB1-bio-His (proTGF $\beta$ ) which was a gift from Gavin Wright (Addgene plasmid \# 52185) [17] and HA-OVOL2 (OVOL2) expressing plasmid was a kindly gift from Changwon Park [18]. EMT/MET in VRCs was studied with the use of expressing vectors harbouring genomic fragments of microRNA-200 family (miR-145, miR-200b, miR-200c, miR-205) which were cloned in our laboratory (See Molecular Cloning section). Control cells were transfected with pUC18 sub-cloning plasmid.

\subsection{Molecular cloning}

All the plasmid fragments used for cloning were amplified using tiHybrid proofreading DNA polymerase (EURx), according to the supplied protocol. PCR products amplified on the plasmid DNA template were incubated overnight at $37{ }^{\circ} \mathrm{C}$ with DpnI FastDigest enzyme (Thermo) as in manufacturer's instructions.

VIM-T2A-mCardinal sequence was cloned from cDNA of knocked-in cells upon PCR amplification into linearized by PCR pmR expressing vector (Clonetech) and recombined using Gibson Assembly reagent (NEB). Resulting vector contained full VIM-T2A-mCardinal reading frame under the control of CMV promoter. The primers for insert amplification were KI-F and KI-R whereas the pair used for backbone linearization were BCB-F and BCB-R (Supplementary data, Table 1). Mutagenesis was performed by REPLACR methodology [19], using the SDM-F and SDM-R primers (Suppl material Table 1).

The vectors harbouring miR-145, miR-200b, miR-200c and miR-205 genomic fragments were created by inserting each PCR amplified microRNAs gene into the $3^{\prime} \mathrm{UTR}$ of mNeon fluorescent protein expressing vector (pmR-mNeon). All listed above genomic fragments were amplified using tiHybrid DNA polymerase (EURx) from the DNA, which was purified from the blood of healthy volunteer with the use of GeneAll Exgene Blood SV kit (GeneAll). The sets of primers used for amplification of miR-145, miR-200b, miR-200c and miR-205 fragments were miR145-F/miR145-R, miR-200b-F/miR200b-R, miR-200c-F/ miR-200c-R, miR-205-F/ miR-205-R respectively (Suppl material Table 1.). The amplified products produced proper sticky ends upon digestion by BgIII and HindIII restrictases (both from Thermo). Digested and purified DNA fragments were ligated using T7 ligase (Thermo) in molar ratio 3:1 with $100 \mathrm{ng}$ of linear pmR-mNeon, which was previously cut by BglII and HindIII enzymes.

Resulting vectors were named miR-145, miR -200b, miR-200c, miR-205. The sequences of all the vectors were verified by Sanger sequencing (Genomed).

\subsection{Genotyping}

The targeting sequence for CRISPR/Cas9 was in the last intron (intron 8) of VIM in HEK293 and H2170 cells with the use of Benchling algorithm. Single-cell clones were cultured on 96-well plates to obtain more than $50 \%$ of confluence (Nunc). Upon washing with Phosphate-buffered saline (PBS, Gibco) 
121 they were genotyped by PCR using Mouse Direct PCR Kit (Bimake), following the manufacturers

122 instruction. The primers used for genotyping were 170 and 249 (Supplementary data, Table 1).

123 The genotyping was further confirmed by PCR using the same set of primers (170 and 249), tiHybrid

124 DNA polymerase and high quality genomic DNA, purified from single-cell clones with the use of 125 QIAamp DNA Mini Kit (Qiagen). Then the KI was verified by sequencing (Genomed).

\subsection{RNA extraction, reverse transcription and $q P C R$}

127 Total RNA was isolated from cells with the use of Extractme Total RNA kit (Blirt) according to 128 manufacturer's manual, including DNase treatment. The purity and quantity of isolated RNA was 129 estimated spectrophotometrically with the use of Tecan M200Pro microplate reader supplied with 130 NanoQuant plate (Tecan). Only the samples with 260/280 nm OD ratio higher than 1.8 were used for 131 downstream analysis. For molecular cloning $3 \mu \mathrm{g}$ of RNA were reverse transcribed for $30 \mathrm{~min}$ at $50^{\circ} \mathrm{C}$ 132 using an oligo(dT) primer and the Transcriptor High Fidelity cDNA Synthesis Kit (Roche) followed 133 by 5 min enzyme inactivation at $85^{\circ} \mathrm{C}$ according to manufacturer's instructions. For QPCR, $2 \mu \mathrm{g}$ of 134 RNA were reverse transcribed using High-Capacity RNA-to-cDNA Kit (Applied Biosystems) 135 following to the manufacturer's protocol.

136 Quantitative real-time expression analysis was performed using a LightCycler ${ }^{8} 80$ II instrument 137 (Roche) equipped with 384 well plates and PowerUp ${ }^{\text {TM }}$ SYBR $^{\text {TM }}$ Green Master Mix (Applied 138 Biosystems). The primers were as listed in Supplementary data (Table S1). Amplification was 139 performed in $12,5 \mu \mathrm{l}$ reaction mixture containing cDNA amount corresponding to $12.5 \mathrm{ng}$ of total 140 RNA, $1 \times$ PowerUp SYBR Green Master Mix and 3.125 pmol of each primer (Forward and Reverse).

141 Upon $2 \mathrm{~min}$ of initial incubation at $50{ }^{\circ} \mathrm{C}$ followed by 2 min incubation at $95^{\circ} \mathrm{C}$, cDNA was amplified 142 in 45 cycles consisting of $15 \mathrm{~s}$ denaturation at $95^{\circ} \mathrm{C}, 30 \mathrm{~s}$ annealing at $60^{\circ} \mathrm{C}$ and $20 \mathrm{~s}$ elongation at $72^{\circ} \mathrm{C}$. 143 Obtained fluorescence data was analyzed using a relative quantification (RQ) method $2^{\wedge}(-\Delta \Delta \mathrm{CT})$ for 144 estimating expression fold changes normalized to dim-VRCs and $2^{\wedge}(-\Delta C T)$ method for comparison 145 of the expression of each measured gene. The assessed genes expression (VIM, mCardinal, CDH1, 146 ZEB1 and ZEB2) were normalized to GAPDH level, which was measured with the use of GAPDH-F 147 and GAPDH-R oligonucleotides. GAPDH was previously confirmed as stably expressed at mRNA 148 level in $\mathrm{H} 2170$ as well as in VRCs (mean $\mathrm{Cp}=17.14$; median $\mathrm{Cp}=17.09$; $\mathrm{SD}=0.5209$; $\mathrm{SEM}=0.03638$; $149 \mathrm{~N}=205$ ). Expression of VIM was measured using VIM-F and VIM-R primers, measurement of $150 m$ Cardinal level was conducted using mCard-F and mCard-R primers, whereas estimation of CDH1 151 expression was performed at mRNA level with the use of Cdh1-F and Cdh1 -R oligonucleotides. 152 ZEB1 and ZEB2 quantifications were performed using ZEB1F/R and ZEB2F/R pairs of 153 oligonucleotides, respectively. TWIST1 and TWIST2 quantifications were performed using 154 TWIST1F/R and TWIST2F/R pairs of oligonucleotides, respectively. The primers were designed as 155 intron-spanning to avoid any influence of genomic DNA contamination and was listed in 156 Supplementary materials (Table S1).

$157 \quad 2.7$ Flow cytometry of living cells

158 VRCs or HT29 cells cultured for one day on 6 well plate (Nunc) were washed with HBSS - Hank's 159 Balanced Salt Solution (Thermo) and detached by Accutase (Corning). The cells dissolved in $100 \mu \mathrm{l}$ 160 HBSS with $5 \mu$ l of PE Mouse anti-E-Cadherin (562526, BD Pharmingen) were incubated for 1 hour, 
161 washed with HBSS and suspended in $0.5 \mathrm{ml}$ of HBSS. The cells were counted on FL-3 fluorescence

162 channel using FACSCalibur flow cytometer (BD).

$163 \quad 2.8$ Cell sorting

164 VRCs seeded one day before were then sorted using the BD FACSAria ${ }^{\mathrm{TM}}$ flow cytometer (BD Biosciences). In 165 this case, cells were selected into two populations due to the intensity of their mCardinal fluorescence in far166 red channel (Figure S1). After sorting, the purity of sorted cells was confirmed by flow cytometry and reaches 167 more than $97 \%$. The resulting two populations of VRCs were named dim-VRCs and bright-VRCs.

\section{2.9 Confocal imaging of living cells}

169 The cells were seeded onto 24-well glass-bottom plates (MoBiTec) a day before transfection.

170 Transfection was conducted as described above. Transfected cells were then visualized under Nikon

171 Ti Confocal microscope in 24 and $28 \mathrm{~h}$ upon transfection using 563nm laser for mCardinal

172 fluorescence. Mean fluorescence intensity was measured using NISelements (ver 3.22.08) software

173 and shown on the graphs. Each of at least 10 measured Regions of the interest (ROIs) included the 174 cluster of more than 20 adherent cells. The brightest slice of the z-stack were chosen for the 175 measurement. The measurement were done in duplicate.

\subsection{Immunocytochemistry}

177 The cells seeded a day before on glass bottom Labtec chamber slides (Nunc) were washed with PBS 178 and fixed with $2 \%$ paraformaldehyde for 20 minutes in room temperature. Upon washing in PBS the 179 cells were permeabilized by $0.5 \%$ triton in PBS for $20 \mathrm{~min}$. The endogenous peroxidase was blocked 180 by incubation in 1\% sodium azide and 1\% hydrogen peroxide in PBS for another $20 \mathrm{~min}$. Another 181 washing step in PBS was followed by incubation in Blocking Buffer which was 1x Normal Donkey 182 Serum (reconstituted from 20x, Jackson ImmunoResearch) containing 1\% bovine serum albumin 183 (SantaCruz Biotechnology) and $0.1 \%$ of triton for $30 \mathrm{~min}$ in room temperature. Supplied by Cell 184 Signaling primary antibodies against VIM (rabbit, \#5714S) and FLAG-tag (mouse, \#8146S) were 185 diluted in Blocking Buffer in ratio 1:100 and 1:1600 respectively. Incubation with the primary 186 antibodies was conducted overnight at $4^{\circ} \mathrm{C}$ and was followed by washing in PBS. Secondary 187 Peroxidase $\mathrm{F}\left(\mathrm{ab}^{\prime}\right)_{2}$ Fragment Donkey Anti-Rabbit IgG (H+L) (711-036-152, Jackson ImmunoResearch) 188 for detection of primary rabbit and Peroxidase $\mathrm{F}\left(\mathrm{ab}^{\prime}\right)_{2}$ Fragment Donkey Anti-Mouse IgG (H+L) (715189 036-150, Jackson ImmunoResearch) for detection of primary mouse antibodies were diluted in 190 Blocking Buffer 1:1000 respectively. Incubation with secondary antibodies (fluorochrome or 191 peroxidase conjugated) was occurred for $1 \mathrm{~h}$ in room temperature and was followed by another 192 washing in PBS. Peroxidase conjugated antibody were stained with Alexa Fluor 555 Tyramide 193 Reagent (B40955, LifeTechnolgies) or Alexa Fluor 488 Tyramide Reagent (B40953, LifeTechnolgies) 194 following to the manufacturers protocol, resulting in enzyme-linked fluorescence signal 195 amplification. When double Alexa Fluor Tyramide Reagents (488 and 555) staining was done an 196 additional step, 15 minutes washing in 3\% hydrogen peroxide, $0.1 \%$ sodium azide in PBS, to achieve 197 complete inhibiting of HRP enzyme was added between incubations with two different secondary 198 HRP-antibodies. The cells were finally washed by PBS and stained with Hoechst (Cayman) diluted 199 in PBS (1:1000 from $10 \mathrm{mg} / \mathrm{mL}$ stock solution). The cells were washed once again, mounted with 
200 ProLong Gold mounting medium (LifeTechnologies) and visualized under Nikon Ti confocal

201 microscope.

202 2.11 Migration of VRCs

203 XCELLigence real-time cell analysis system (Acea) equipped with 16-wells CIM-plates served for 204 VRCs migration assessment. VRCs cells first detached using Accutase, then transfected with OVOL2, 205 miR-200c, miR-205 or pUC18 plasmids using Lipofectamine300 reagent, as was described above. 206 Then, 30,000 of cells were seeded on each well of the CIM-plate, kept in the standard mammalian 207 tissue culture conditions and measured for 24 hours. The cell migration toward an attractant, which 208 was 10\% DMEM supplemented with 10\% FCS was normalized to cell migration toward DMEM alone 209 and shown as Cell Index. Each experiment was measured at latest in triplicates and repeated twice.

\section{3. Results}

\section{3.1.1 Genome editing}

212 The targeting sequence in H2170 cells was confirmed by sequencing (Figure S1). The H2170 lung 213 cancer cells were nucleofected with the CRISPR and template (vKIT, Figure 1A) plasmids, following 214 enrichment using puromycin. vKIT contained the sequence of self-cleaving T2A peptide followed by 215 sequence of FLAG-tagged mCardinal fluorescent protein gene, flanked by two 800 nucleotides long 216 homology arms (HA) that corresponds to VIM gene fragments near CRISPR-targeting region (Figure 217 S2).

218 The template cassette, containing HA-T2A-FLAG-mCardinal-HA, was flanked by two gRNA targeting 219 sequences identical to those for VIM gene in order to linearize the knock-in insert and promote 220 efficient Homology directed repair (HDR).

221 Two days after nucleofection, the cells were selected using puromycin $(1 \mu \mathrm{g} / \mathrm{ml})$ for another two days. 222 Single cell clones were obtained by dilution, further they were genotyped by PCR with the efficiency 223 near 2,7\% (3/112). Single cell clones, confirmed by PCR, were called VRCs and used in downstream 224 analysis. The sequence of knocked-in DNA fragment in the VRCs was further verified by Sanger 225 sequencing (Figure S1).

226 The subcellular localisation of mCardinal in living cells (Figure 1B-D) by confocal imaging showed 227 that mCardinal was present in the cytoplasm, but its concentration was not homogenous showing 228 bright foci as well as cytoplasmic localisation. Immunofluorescent labelling of VIM and FLAG229 mCardinal (Figure 2A-F) shows almost complete co-localization, where VIM was found in the 230 cytoskeleton as well as in the foci.

231 The unexpected cellular localization of VIM made us to analyze if the gene product was intact. For 232 this we amplified the entire VIM-T2A-mCardinal from mRNA, and cloned it into an expression vector. 233 The whole VIM-T2A-mCardinal sequence was sequence-verified. This VIM-T2A-mCardinal cDNA 234 exerted identical cellular distribution of mCardinal under the confocal microscope when expressed 235 in HEK293 cells (Figure S3 A-C).

236 To determine if fusing mCardinal to VIM would result in a better cellular distribution, the T2A 237 peptide sequence was modified by substitution of the P16A and P18A in the T2A sequence. The 238 resulting vector (VIM-mCardinal) was overexpressed in HEK293 cells, showing granular localisation 
too (Fig S3 D-F). Overexpression of VIM-mCardinal in HEK293 cells showed that this fusion protein localised only in large granules in the cell and is similar in comparison to the fluorescence in VIMT2A-mCardinal overexpressing cells, where the fluorescence was shown in similar granules as well as diffusing in the cytoplasm (Figure S3).

Since the foci in the Vim-T2A-mCardinal cells corresponded to fused Vim-mCardinal fusions, likely resulting from inefficient T2A processing, were brighter than if evenly distributed throughout the cytoplasm, we considered them as a baseline.

To further characterise VRCs, they were then sorted according to the fluorescence intensity, resulting in two populations (dim-VRCs and bright-VRCs) that differ about 3 times in mCardinal fluorescence intensity (Figure S4). That difference was reduced upon culturing to less than 2 fold mCardinal intensity between dim-VRCs and bright-VRCs after $48 \mathrm{~h}$ (Figure 2G). VIM as well as mCardinal expression measured at the transcriptional level confirmed that those two genes are expressed at equal levels (Figure 2H and S5).

The dim-VRCs where then exposed to a series of EMT-inducing factors, to analyse the correlation between mesenchymal conversion and mCardinal expression.

\subsubsection{EMT markers vs reporter gene}

The dim-VRCs were transfected with active Snai1 or proTGF $\beta$ expressing plasmids, which resulted in a 2-fold increase in fluorescence, examined using confocal microscopy in $24 \mathrm{~h}$ or in $48 \mathrm{~h}$ after transfection (Figure 3A). Correspondingly, the transcriptional levels of VIM as well as mCardinal showed similar fold increase (RQ) (Figure 3B-C). The highest expression of VIM was seen in the cells that overexpressed active Snai1 one and two days upon transfection (median RQ $=1.625$ and $R Q=$ 1.580 respectively). proTGF $\beta$ overexpressing dim-VRCs for $24 \mathrm{~h}$ and $48 \mathrm{~h}$ also overexpressed VIM at median level $\mathrm{RQ}=1.371$ and $\mathrm{RQ}=1.424$. Control population of bright-VRCs expressed VIM at median $\mathrm{RQ}=1.569$. The highest level of $m$ Cardinal was expressed in active Snai1 expressed dim-VRCs after $24 \mathrm{~h}$ (median $\mathrm{RQ}=1.357$ ) and in $48 \mathrm{~h}$ after transfection (median $\mathrm{RQ}=1.240$ ). proTGF $\beta$ overexpressing $\mathrm{dim}-\mathrm{VRCs}$ for $24 \mathrm{~h}$ and $48 \mathrm{~h}$ also overexpressed mCardinal at median level $\mathrm{RQ}=1.206$ and $R Q=1.248$, whereas bright-VRCs $m$ Cardinal median RQ was 1.181. The expression levels of VIM as well as $m$ Cardinal relative to GAPDH housekeeping gene show a similar pattern (Figure 3B-C). Epithelial marker $C D H 1$ transcript in proTGF $\beta$ over-expressing dim-VRCs did not reveal significant changes in $24 \mathrm{~h}$ nor in $48 \mathrm{~h}$ upon transfection (Figure 3D). We did not observed significant changes CDH1, VIM, SNAI1, ZEB1, ZEB2, TWIST1 or TWIST2 level between VRCs and parental H2170 cells by qPCR (Figure S6).

\subsubsection{OVOL2 and miRNAs overexpression modulates CDH1 expression in VRCs via ZEB1/2 repressors}

In order to test if mesenchymal-like VRCs could reverse their phenotype into more epithelial, we transfected these cells with a strong epithelial activator - OVOL2 [20]. Immunostaining followed by flow cytometry analysis as well as qPCR confirmed our hypothesis showing that E-cadherin level is elevated in OVOL2 overexpressing VRCs after 48h (Figure 3E). OVOL2 overexpressing VRCs concomitantly showed a significant increase of the mCardinal fluorescence (Figure 3F). To examine the mechanism of epithelial phenotype development by VRCs, we analysed the expression level of CDH1, as well as its negative regulators ZEB1 and ZEB2 (Figure 4A-C) in the cells that overexpressed OVOL2 or four different ZEB1/2-targeting members of the miR-200 family (miR-145, miR-200b, miR- 
200c, miR-205). Both negative regulators of $C D H 1$ expression (ZEB1 and ZEB2) were significantly downregulated in VRCs that overexpressed OVOL2 or miR-205 with simultaneous upregulation of CDH1. miR-200c overexpression in VRCs produced increased level of CDH1 together with downregulated ZEB1, whereas the effect of miR-145 overexpression was seen only as elevated CDH1 level. We did not observe changes in CDH1 or ZEB1/2 levels in mirR-200b-expressing VRCs.

\subsubsection{OVOL2 and miR-200c or miR-205 overexpressing VRCs show decreased migratory potential}

In order to examined if decreased levels of ZEB1/2 could repress migratory properties of VRCs $[20,21]$, the cells were transfected with OVOL2, miR-200c and miR-205 overexpressing plasmids and real time migration assay was conducted. We observed over two fold decrease in the number of migrating VRCs that overexpressed all of tested factors that may modulate ZEB1/2 (OVOL2, miR$200 \mathrm{c}$ and miR-205) in comparison to the mock transfected cells (Figure 4D).

\subsection{Figures}
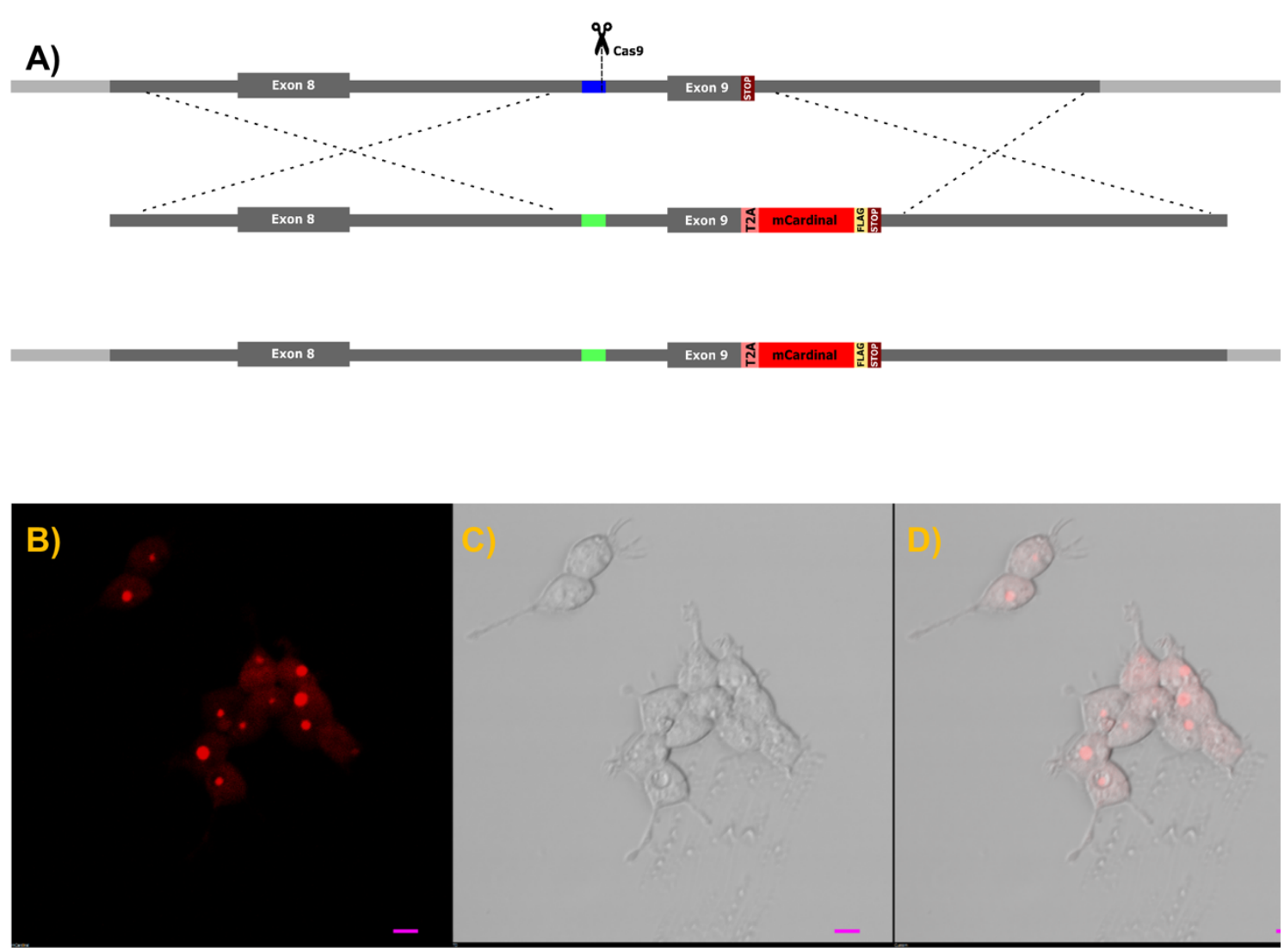

Figure 1. A) Schematic representation of genome editing strategy. Genomic region of H2170 cells targeted by CRISPR/Cas9 with homology arms marked in grey while targeted site in intron 8 has been marked in dark blue. The donor DNA template contains the sequence of T2A-mCardinal-FLAG, flanked by two homology arms that corresponds to VIM gene fragments (grey) recombines with targeted gene. VIM allele in VRCs contains the knocked-in DNA and changed sequence recognized by Cas9 sequence is coloured in green. B-D). Subcellular localization of $\mathrm{mCardinal} \mathrm{fluorescent} \mathrm{protein}$ in living VRCs. The scale bar is $10 \mu \mathrm{m}$. B) mCardinal, C) transmission, D) merged. 


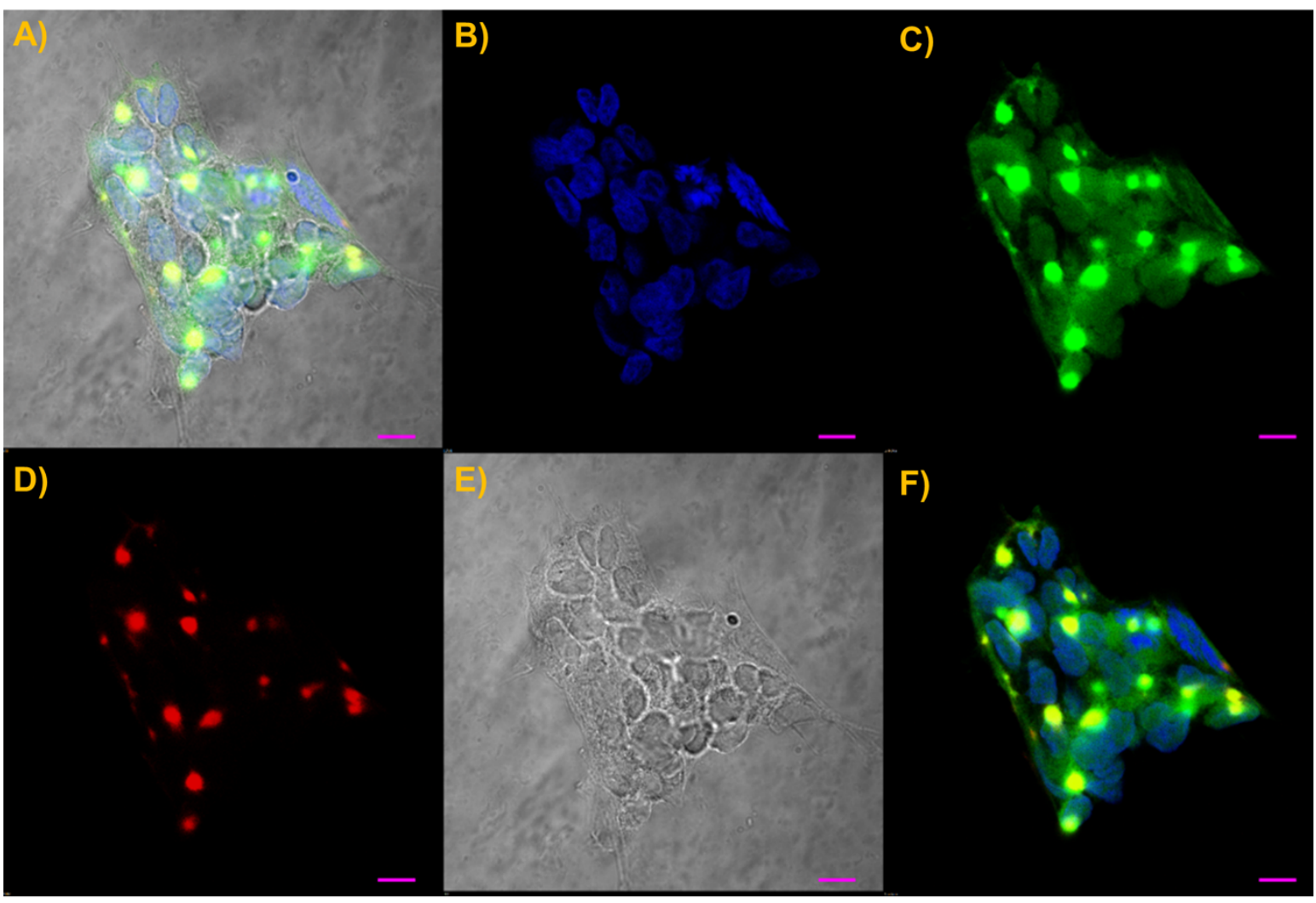

G) dim-VRCs vs bright-VRCs
H)

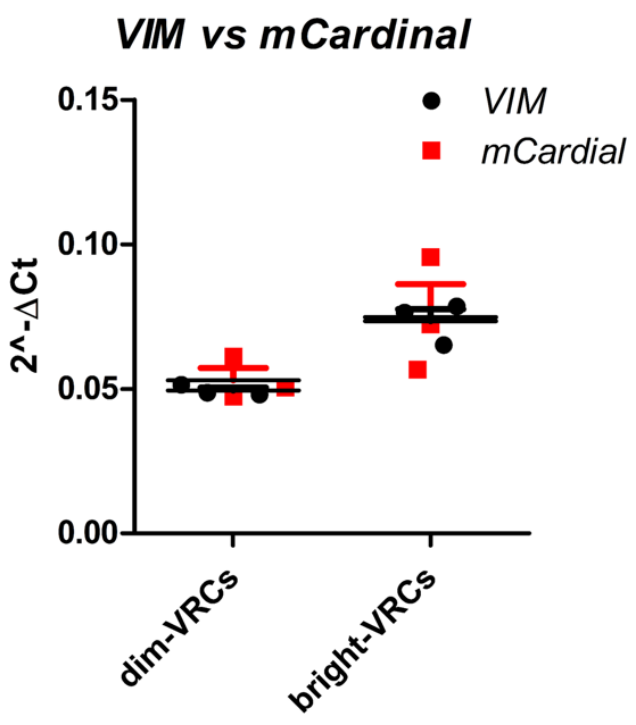

Figure 2. A-F) Immunofluorescent labeling of FLAG-tagged mCardinal and VIM in VRCs. Shown in green FLAG tag (C) is seen as bright spot-like areas surrounded by less intensive fields localized in the cytoplasm. Localization of VIM shown in red (D) corresponds to the regions with the highest fluorescence of FLAG-tagged mCardinal (C). Blue coloured DAPI, FLAG, VIM and transmission were merged on picture A). B) DAPI, C) anti-FLAG, D) VIM, E) transmission, F) merged fluorescence. The scale bar is $10 \mu \mathrm{m}$. G) mCardinal fluorescence changes of sorted VRCs populations with time. The fluorescence of dim-VRCs and bright-VRCs populations was measured in $24 \mathrm{~h}$ and $48 \mathrm{~h}$ of culture upon sorting. The points correspond to the fluorescence of selected ROI, whereas the lines show mean fluorescence. The representative results was shown. H) VIM and $m$ Cardinal quantification by qPCR shows similar amount of both transcripts. VIM and mCardinal genes were measured in dim-VRCs transfected cells in 24 or $48 \mathrm{~h}$ upon transfection. The data shows mean $2^{\wedge}(-\triangle \mathrm{CT})$ relative to GAPDH 
311 housekeeping gene. The graph shows the representative result of the measurement which was done 312 in triplicate.

313
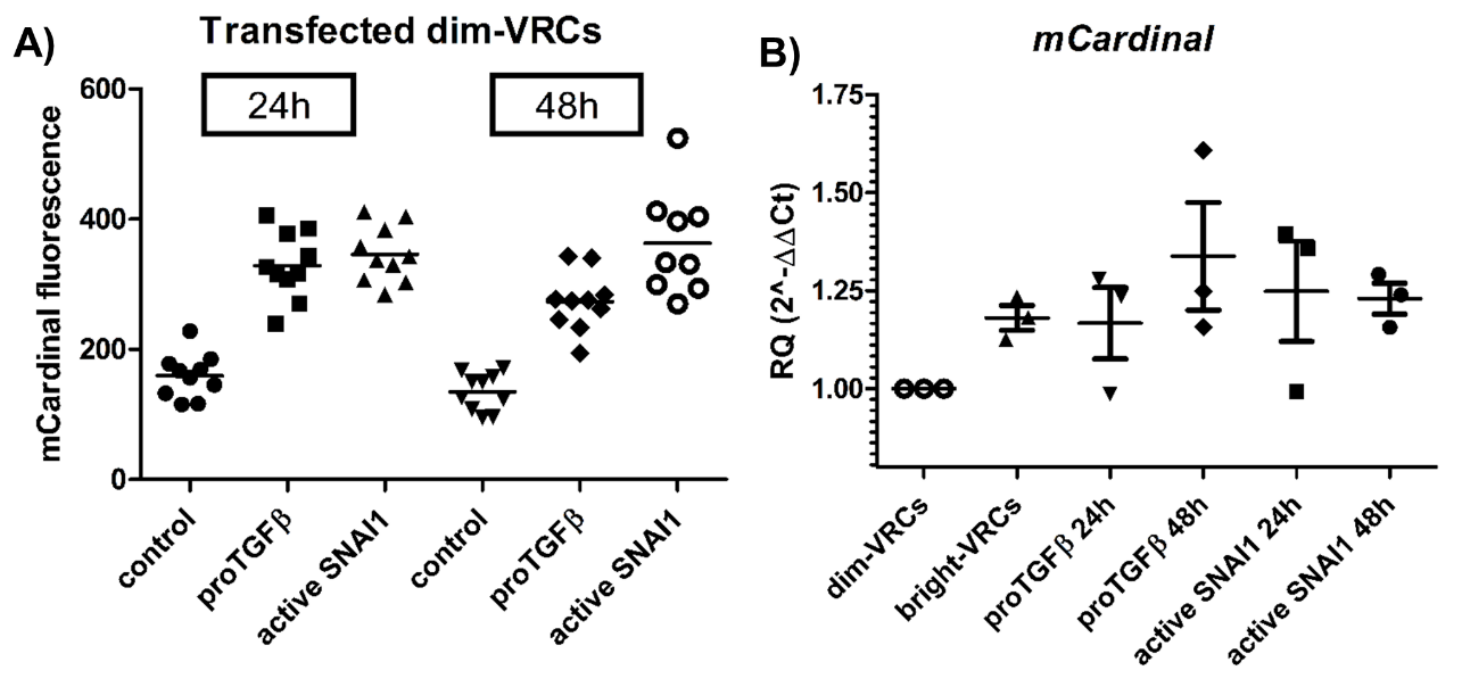

D) $\quad \mathrm{CDH1}$

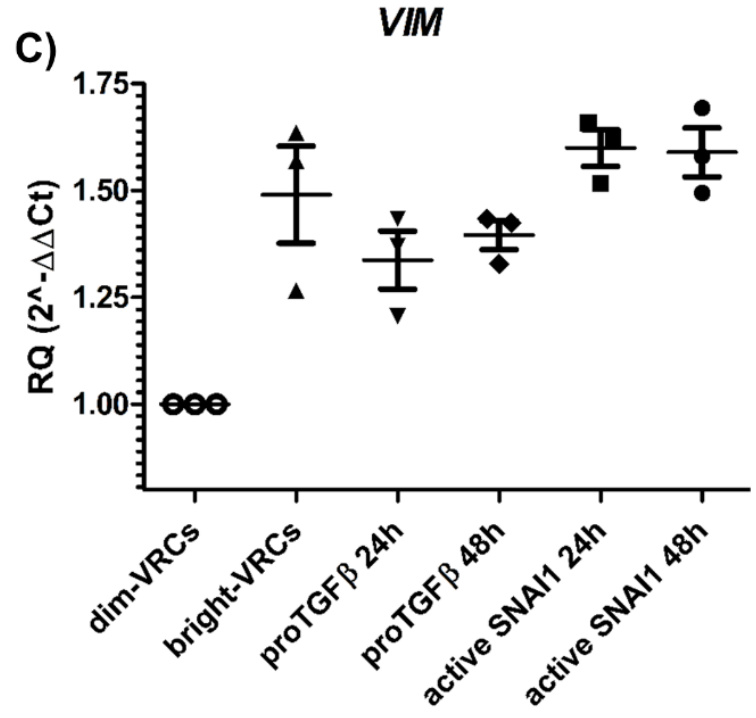

E)

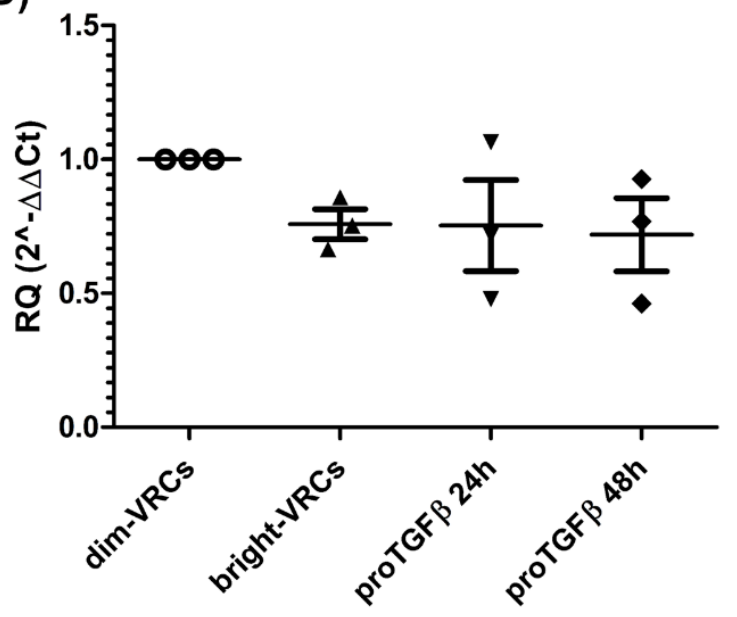

F)

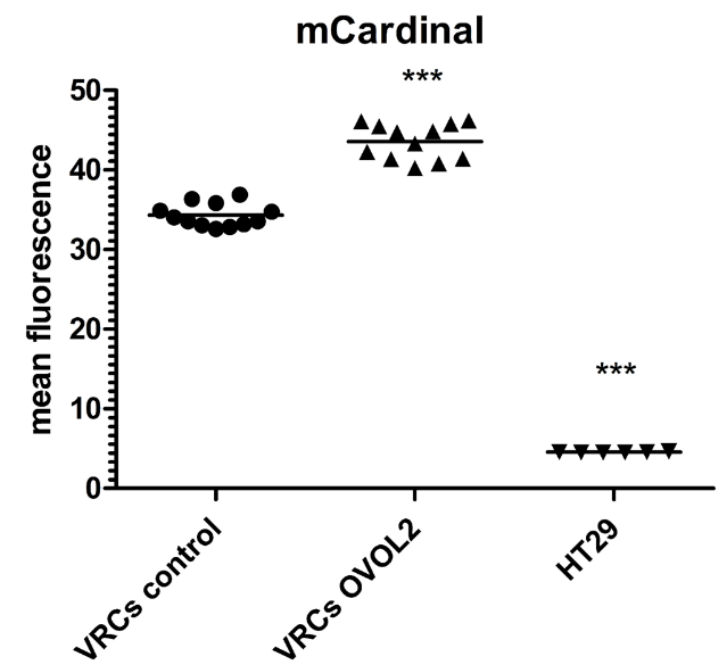

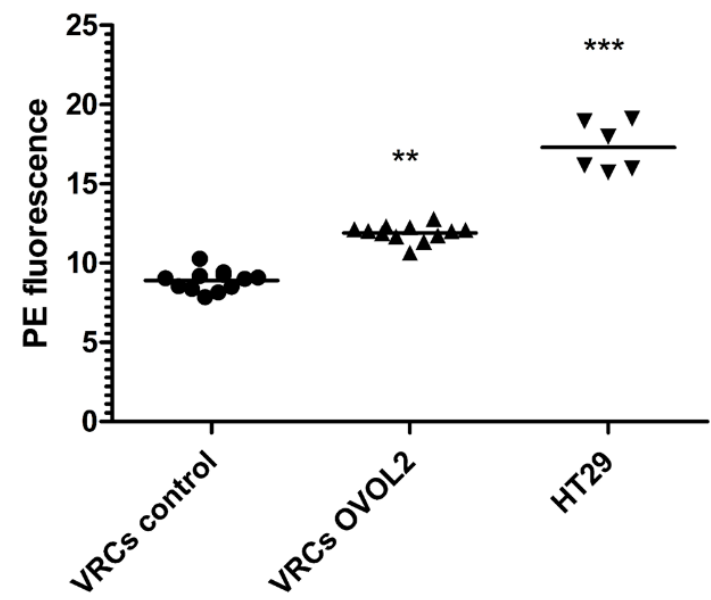

Figure 3. A) mCardinal fluorescence of dim-VRCs population transfected with active SNAI1 or proTGF $\beta$ plasmids. The fluorescence of the cells was measured in $24 \mathrm{~h}$ and $48 \mathrm{~h}$ of culture upon sorting. The points correspond to the fluorescence of selected ROI, whereas the lines show mean 
(RQ) by qPCR. VIM and mCardinal genes were measured in dim-VRCs transfected cells in 24 or 48 $\mathrm{h}$ upon transfection. The data shows mean $2^{\wedge}(-\triangle \Delta C T)$ relative to GAPDH housekeeping gene. The results were normalized to control which were pUC18 transfected dim-VRCs cells. The graph shows the representative result of the measurement which was done in triplicate. D) Relative quantification of $C D H 1$ by qPCR. CDH1 levels were measured in proTGF $\beta$ transfected dim-VRCs in 24 or $48 \mathrm{~h}$ upon transfection. The graph shows mean $\mathrm{RQ} 2^{\wedge}(-\Delta \Delta \mathrm{CT}) \pm$ SEM relative to GAPDH housekeeping gene. The results were normalized to control which were pUC18 transfected dim-VRCs cells $48 \mathrm{~h}$ upon transfection. The graph shows the representative result of the measurement which was done in triplicate. No effect in CDH1 expression. E) Immunostaining of E-cadherin and F) mCardinal fluorescence in VRCs and HT29 E-cadherin positive cells by flow cytometry. The graph shows: VRCs control were VRCs transfected with pUC18 vector whereas, VRCs OVOL2 were OVOL2 overexpressing in $24 \mathrm{~h}$ upon transfection. Immune-stained un-transfected HT29 cells were used as controls. The graph shows the single reads as well as the mean value of 3 independent experiments. Statistical significance in comparison to control was calculated using Mann-Whitney test and was rated by asterisk: ${ }^{*} \mathrm{p}>0.05 ;{ }^{* *} \mathrm{p} 0.01 ;{ }^{* * *} \mathrm{p}<0.01$.

A)

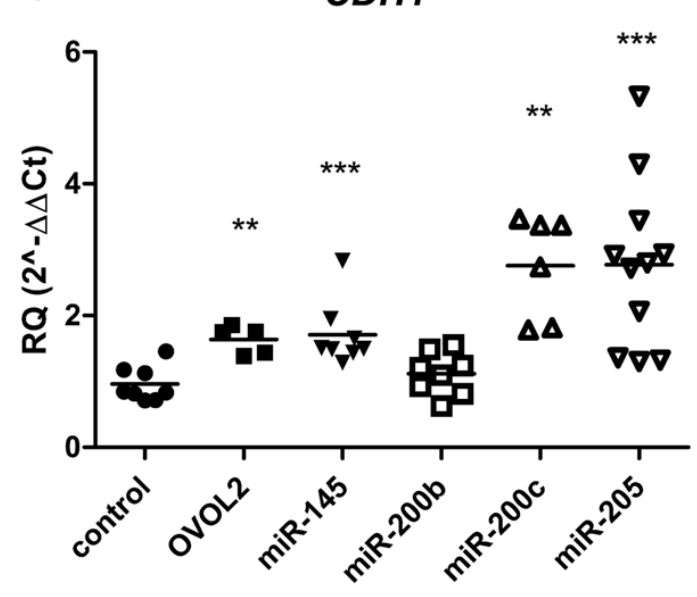

C)

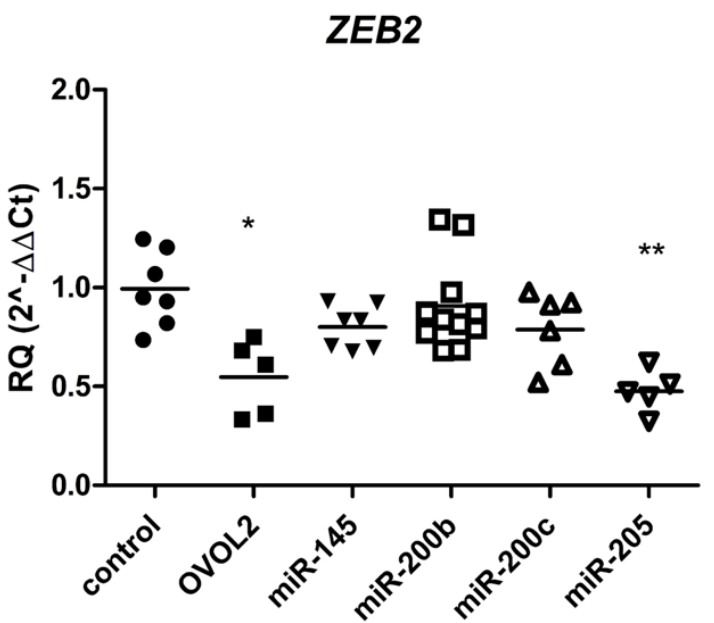

B)

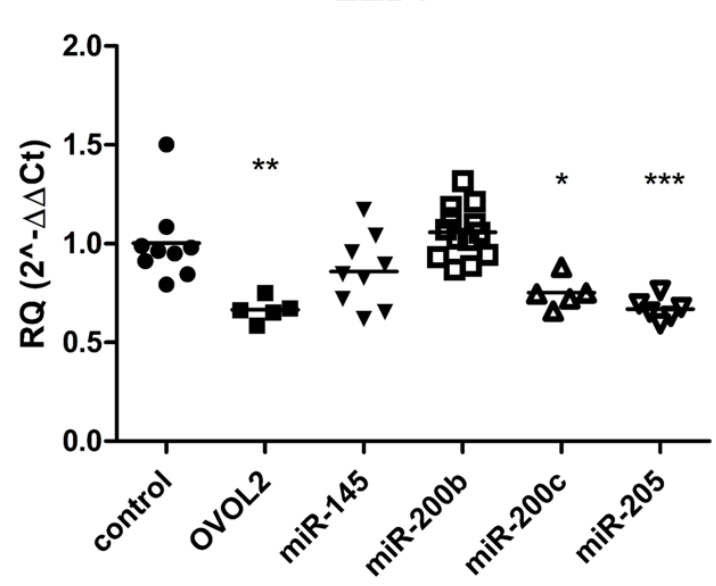

D)

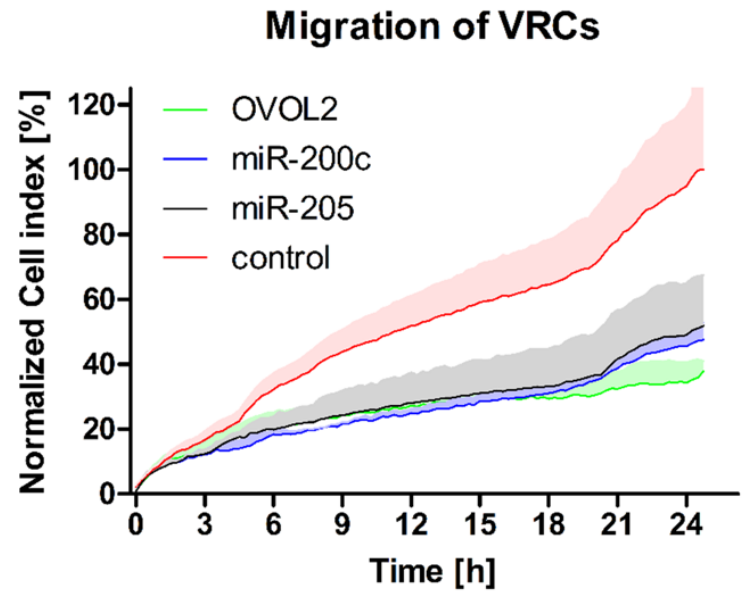

Figure 4. A-C) Relative quantification of $C D H 1, Z E B 1$ and $Z E B 2$ transcripts in transfected VRCs by qPCR. The expression of genes relative to GAPDH housekeeping gene was measured in $48 \mathrm{~h}$ upon transfection of VRCs with OVOL2 and microRNA expressing vectors (miR-145, miR-200b, miR-200c 
$\mathrm{RQ} 2^{\wedge}(-\Delta \Delta \mathrm{CT})$ as well as single values. The graph contains data from at least two independent experiments which were measured in triplicate. Statistical significance in comparison to control was calculated using Mann-Whitney test and was rated by asterisk: ${ }^{*} p>0.05 ;{ }^{* *} p 0.01 ;{ }^{* * *} p<0.01$. D) Realtime migration analysis of transfected VRCs using xCELLigence system. The line shows mean normalized cell index, whereas coloured area visualizes standard deviation of 3 replicates. Migration of VRCs transfected with: OVOL2 is shown in green, miR-200c shown in blue, miR-205 shown in black, whereas pUC18 as the control is coloured in red. The representative results from two different experiments was presented.

\section{Discussion}

Mounting evidence shows that tumors are far more heterogenous than expected in due to genetic diversity of the tumor cells as well as their phenotypic plasticity [22,23]. This phenotypic plasticity is in fact a phenotype switching, what is understood as a phenomenon whereby cancer cells transit between different phenotypes in response to environmental cues, without acquiring new mutations [24]. This cellular plasticity has been reported as having high clinical impact because it is crucial for drug resistance development e.g. in lung cancer patients treated with EGFR inhibitors [25], reacquiring pluripotency and become a cancer stem cell (CSC) [26], or maintaining metastatic ability of many types of cancer cells [27-29].

Among tumor cells there are some that undergo EMT, MET, or hybE/M, and in order to understand the effects of cellular plasticity in biological and pathological processes, there is a need of reporters that can determine the stage of a cell. Observing changes in mesenchymal and epithelial phenotype as they occur, is a significant improvement in studying of molecular mechanism in cancer cells. H2170 cells was chosen because they have been a good model for to EMT and MET [30,31].

To-date EMT/MET is routinely studied by the use of exogenous reporter genes that are heterologously expressed exhibiting interference of cis- and trans-regulatory elements, alternative promoters or epigenetic events that resulted in obtaining unreliable data [32,33]. These limitations are overcome by using genome engineering of endogenous genes. There are a couple of available cancer cell lines that harbor a C-terminal red fluorescent protein (RFP) tag on VIM: A549 (lung), HCT116 (colorectal), MDA-MB-231 (breast adenocarcinoma) and they are commercially available at ATCC cell repository. These cells however, have the RFP knocked-in into the beginning of last exon of VIM (exon 9), what results in deletion of large fragment of that exon and of the gene product (https://www.lgcstandards-atcc.org/en/Global/Products/CCL-247EMT.aspx\#documentation). While, these modified A549, HCT116 or MDA-MB-231 cell lines enable near real-time tracking of the EMT/MET status as cells transition from epithelial to mesenchymal phenotype under defined conditions [34], any approach where the an endogenous protein is truncated may affect functions in comparison to endogenously expressed proteins, , in particular in the context of a protein such as VIM which has many signaling modulatory activities during cell plasticity [35-37].

Using a far-red fluorescent protein (mCardinal) which is simultaneously expressed with endogenous VIM, but separate during translation due to the viral self-cleaving peptide (T2A). T2A was chosen because, together with $\mathrm{P} 2 \mathrm{~A}$, it have been reported as the most efficient from all tested self-cleaving 2A peptides [38-41], moreover T2A resulted in the least amount of "uncleaved" protein product among the family of 2A peptides [39-41]. The stability and half-life of the 2 resulting proteins can result in small changes in total expression, the two proteins are synthesized at a 1:1 ratio [41]. VIM 
expression at the transcriptional level corresponded to that of mCardinal, and when stimulated, the changes of VIM expression when hand-to-hand to those of $m$ Cardinal, evidencing that our strategy is fully functional.

mCardinal was chosen by us mainly because this far-red monomeric protein is better for in vivo settings due to it is excitation as wavelengths above $600 \mathrm{~nm}$, which penetrate through hemoglobinrich tissues far better than lower wavelengths [42]. The second reason is that the mCardinal is suitable for precise monitoring of its changing expression in due to its short maturation half-time (27 min) [43] which is about 3 times shorter when we compare others commonly used fluorescent markers e.g. RFP [44].

The subcellular localisation of mCardinal in VRCs by confocal imaging show non-homogenous distribution of the fluorescence which was observed at moderate level in cytoplasm as well and in brighter glowing foci. Immunofluorescent staining of VIM and FLAG-tag mCardinal showed colocalization at the "foci", whereas Vimentin is also present in the cytoskeleton of VRCs. Moreover, re-cloning of and the VIM-P2A-mCardinal and further over-expressing it in HEK293 cells shown similar sub-cellular distribution of the fluorescence as in VRCs. The presence of foci is probably the result of not fully efficient T2A peptide is cleavage, the fusion protein being unable to form normal VIM polymers [39-41], which is more conspicuous in the case of the fusion of VIM-mCardinal. In fact, C-tags in VIM might result in an impaired protein [45], and it's expression may give dominantnegative phenotype, similar to the phenotype observed in the cells which express only the N-terminal domain of VIM (NTD-VIM) [46]. NTD-VIM domain (VIM that lacks C-terminus) expressing cells show its abnormal localization, as formation of the granules in the cytoplasm together with disrupted endogenous VIM localization examined by immunostaining [45]. The role of the tail domain in VIM organisation is not fully understood, although it is known to be necessary for appropriate network formation [47]. Thus, it seems to be possible that abnormal VIM localisation in VRCs may be produced by the fluorescent protein. Yet, the presence of bright foci is advantageous as these structures are brighter than diffused cytoplasmic mCardinal under low VIM expression.

Dim-VRCs presented a reduced mesenchymal phenotype as compared to bright-VRCs. The dimVRCs could be driven to a more mesenchymal phenotype via expression of proTGF $\beta$ [17] or constitutively active SNAI1 transcription factor [16]. We also noticed insignificant downregulation of CDH1 in proTGF $\beta$ over-expressing dim-VRCs, what suggests that E-cadherin may be only partially regulated via TGF $\beta$-dependent pathway [48].

OVOL2 overexpressing VRCs respond by E-cadherin expression, what is in accordance with data describing OVOL2 as a strong epithelial regulator that maintains transcriptional programs in epidermal keratinocytes and mammary epithelial cells by repressing ZEB1 and ZEB2 [49,50].

MiR-200 family were identified as double-negative regulators by silencing $C D H 1$ and transcription repressors Zeb1 and Zeb2 [51,52]. We confirmed the activatory role of miR-145, miR-200c, miR-205 on CDH1 expression in VRCs. We also confirmed that miR-200c expression decreased ZEB1 level in VRCs [53], but we did not observe any effect on miR-200c on ZEB2 expression [54]. VRCs overexpressed miR-205 shown ZEB1/2 downregulation in cancer cells, what is in accordance with previous works $[55,56]$. Our results, suggests that the molecular mechanisms of miR-200 family on EMT might be cell type specific and more work needs to be done to clarify this. Our data is in 
accordance with previous data in that the migratory potential of mesenchymal cells is inhibited by ZEB1 or ZEB2 downregulation produced by miR-200 family overexpression [57].

The very similar level of CDH1, VIM, SNAI1, ZEB1, ZEB2, TWIST1 or TWIST2 transcript between VRCs and parental $\mathrm{H} 2170$ cell line suggests that VRCs may be considered as the model that is useful in broad range of studies.

In conclusion, our data uniquely illustrate a reporter line, VRCs, as a reliable reporter model for studying EMT and MET. VRCs allow to directly observe of cellular plasticity with respect to their mesenchymal/epithelial in vitro and in the future in vivo. These cells could also be used as a robust platform for drug development.

Author Contributions: Conceptualization, ARM, JCz; Methodology, MK, JCz, ARM; Validation, MK, JW; Formal Analysis, MK; Investigation, MK; Resources, AS, ARM; Writing - Original Draft Preparation, MK; Writing - Review \& Editing, MK, JCz, JK; Visualization, MK; Supervision, ARM, AS; Project Administration, ARM; Funding Acquisition, ARM.

Funding: Our work was supported by the Polish National Science Centre (NCN): DEC-2015/17/B/NZ1/01777 and DEC-2017/25/B/NZ4/02364 grants.

Acknowledgments: We would like to thank to prof. Jacek Roliński for enable of the use of his lab equipment.

Conflicts of Interest: The authors declare no conflict of interest.

\section{References}

1. Vicente-Dueñas, C.; Gutiérrez de Diego, J.; Rodríguez, F.D.; Jiménez, R.; Cobaleda, C. The role of cellular plasticity in cancer development. Curr. Med. Chem. 2009, 16, 3676-85.

2. Pastushenko, I.; Blanpain, C. EMT Transition States during Tumor Progression and Metastasis. Trends Cell Biol. 2019, 29, 212-226.

3. Dauphin, M.; Barbe, C.; Lemaire, S.; Nawrocki-Raby, B.; Lagonotte, E.; Delepine, G.; Birembaut, P.; Gilles, C.; Polette, M. Vimentin expression predicts the occurrence of metastases in non small cell lung carcinomas. Lung Cancer 2013, 81, 117-122.

4. Mittal, V. Epithelial Mesenchymal Transition in Aggressive Lung Cancers. In Advances in experimental medicine and biology; 2016; Vol. 890, pp. 37-56.

5. Xiao, D.; He, J. Epithelial mesenchymal transition and lung cancer. J. Thorac. Dis. 2010, 2, 1549.

6. Siegel, R.L.; Miller, K.D.; Jemal, A. Cancer statistics, 2017. CA. Cancer J. Clin. 2017, 67, 7-30.

7. Ruscetti, M.; Quach, B.; Dadashian, E.L.; Mulholland, D.J.; Wu, H. Tracking and Functional Characterization of Epithelial-Mesenchymal Transition and Mesenchymal Tumor Cells during Prostate Cancer Metastasis. Cancer Res. 2015, 75, 2749-2759.

8. Fischer, K.R.; Durrans, A.; Lee, S.; Sheng, J.; Li, F.; Wong, S.T.C.; Choi, H.; El Rayes, T.; Ryu, S.; Troeger, J.; et al. Epithelial-to-mesenchymal transition is not required for lung metastasis but contributes to chemoresistance. Nature 2015, 527, 472-476. 
9. Zheng, X.; Carstens, J.L.; Kim, J.; Scheible, M.; Kaye, J.; Sugimoto, H.; Wu, C.-C.; LeBleu, V.S.; Kalluri, R. Epithelial-to-mesenchymal transition is dispensable for metastasis but induces chemoresistance in pancreatic cancer. Nature 2015, 527, 525-530.

10. Jolly, M.K.; Boareto, M.; Debeb, B.G.; Aceto, N.; Farach-Carson, M.C.; Woodward, W.A.; Levine, H. Inflammatory breast cancer: a model for investigating cluster-based dissemination. npj Breast Cancer 2017, 3, 21.

11. Jolly, M.K.; Ware, K.E.; Gilja, S.; Somarelli, J.A.; Levine, H. EMT and MET: necessary or permissive for metastasis? Mol. Oncol. 2017, 11, 755-769.

12. Toneff, M.J.; Sreekumar, A.; Tinnirello, A.; Hollander, P. Den; Habib, S.; Li, S.; Ellis, M.J.; Xin, L.; Mani, S.A.; Rosen, J.M. The Z-cad dual fluorescent sensor detects dynamic changes between the epithelial and mesenchymal cellular states. BMC Biol. 2016, 14, 47.

13. Jimenez, L.; Wang, J.; Morrison, M.A.; Whatcott, C.; Soh, K.K.; Warner, S.; Bearss, D.; Jette, C.A.; Stewart, R.A. Phenotypic chemical screening using a zebrafish neural crest EMT reporter identifies retinoic acid as an inhibitor of epithelial morphogenesis. Dis. Model. Mech. 2016, 9, 389-400.

14. Gong, S.; Zheng, C.; Doughty, M.L.; Losos, K.; Didkovsky, N.; Schambra, U.B.; Nowak, N.J.; Joyner, A.; Leblanc, G.; Hatten, M.E.; et al. A gene expression atlas of the central nervous system based on bacterial artificial chromosomes. Nature 2003, 425, 917-925.

15. Ran, F.A.; Hsu, P.D.; Wright, J.; Agarwala, V.; Scott, D.A.; Zhang, F. Genome engineering using the CRISPR-Cas9 system. Nat. Protoc. 2013, 8, 2281-308.

16. Zhou, B.P.; Deng, J.; Xia, W.; Xu, J.; Li, Y.M.; Gunduz, M.; Hung, M.-C. Dual regulation of Snail by GSK-3 $\beta$-mediated phosphorylation in control of epithelial-mesenchymal transition. Nat. Cell Biol. 2004, 6, 931-940.

17. Sun, Y.; Vandenbriele, C.; Kauskot, A.; Verhamme, P.; Hoylaerts, M.F.; Wright, G.J. A Human Platelet Receptor Protein Microarray Identifies the High Affinity Immunoglobulin E Receptor Subunit $\alpha($ FceR1 $\alpha)$ as an Activating Platelet Endothelium Aggregation Receptor 1 (PEAR1) Ligand. Mol. Cell. Proteomics 2015, 14, 1265-1274.

18. Kim, J.Y.; Lee, R.H.; Kim, T.M.; Kim, D.-W.; Jeon, Y.-J.; Huh, S.-H.; Oh, S.-Y.; Kyba, M.; Kataoka, H.; Choi, K.; et al. OVOL2 is a critical regulator of ER71/ETV2 in generating FLK1+, hematopoietic, and endothelial cells from embryonic stem cells. Blood 2014, 124, 2948-52.

19. Trehan, A.; Kiełbus, M.; Czapinski, J.; Stepulak, A.; Huhtaniemi, I.; Rivero-Müller, A. REPLACR-mutagenesis, a one-step method for site-directed mutagenesis by recombineering. Sci. Rep. 2016, 6, 19121.

20. Haensel, D.; Sun, P.; MacLean, A.L.; Ma, X.; Zhou, Y.; Stemmler, M.P.; Brabletz, S.; Berx, G.; Plikus, M. V; Nie, Q.; et al. An Ovol2-Zeb1 transcriptional circuit regulates epithelial 
directional migration and proliferation. EMBO Rep. 2019, 20, e46273.

21. Drake, J.M.; Strohbehn, G.; Bair, T.B.; Moreland, J.G.; Henry, M.D. ZEB1 Enhances Transendothelial Migration and Represses the Epithelial Phenotype of Prostate Cancer Cells. Mol. Biol. Cell 2009, 20, 2207-2217.

22. de Sousa, V.M.L.; Carvalho, L. Heterogeneity in Lung Cancer. Pathobiology 2018, 85, 96-107.

23. Testa, U.; Castelli, G.; Pelosi, E. Lung Cancers: Molecular Characterization, Clonal Heterogeneity and Evolution, and Cancer Stem Cells. Cancers (Basel). 2018, 10, 248.

24. Dhillon, A.S.; Tulchinsky, E. FRA-1 as a driver of tumour heterogeneity: a nexus between oncogenes and embryonic signalling pathways in cancer. Oncogene 2015, 34, 4421-4428.

25. Tulchinsky, E.; Demidov, O.; Kriajevska, M.; Barlev, N.A.; Imyanitov, E. EMT: A mechanism for escape from EGFR-targeted therapy in lung cancer. Biochim. Biophys. Acta - Rev. Cancer 2019, 1871, 29-39.

26. Brabletz, T. To differentiate or not - routes towards metastasis. Nat. Rev. Cancer 2012, 12, 425436.

27. Puisieux, A.; Brabletz, T.; Caramel, J. Oncogenic roles of EMT-inducing transcription factors. Nat. Cell Biol. 2014, 16, 488-494.

28. Brabletz, T.; Kalluri, R.; Nieto, M.A.; Weinberg, R.A. EMT in cancer. Nat. Rev. Cancer 2018, 18, 128-134.

29. O'Leary, K.; Shia, A.; Schmid, P. Epigenetic Regulation of EMT in Non-Small Cell Lung Cancer. Curr. Cancer Drug Targets 2017, 18, 89-96.

30. Kurokawa, M.; Ise, N.; Omi, K.; Goishi, K.; Higashiyama, S. Cisplatin influences acquisition of resistance to molecular-targeted agents through epithelial-mesenchymal transition-like changes. Cancer Sci. 2013, 104, 904-911.

31. Nagathihalli, N.S.; Massion, P.P.; Gonzalez, A.L.; Lu, P.; Datta, P.K. Smoking Induces Epithelial-to-Mesenchymal Transition in Non-Small Cell Lung Cancer through HDACMediated Downregulation of E-cadherin. Mol. Cancer Ther. 2012, 11, 2362.

32. Li, M.; Wang, Y.; Liu, M.; Lan, X. Multimodality reporter gene imaging: Construction strategies and application. Theranostics 2018, 8, 2954-2973.

33. Nowakowski, A.; Andrzejewska, A.; Boltze, J.; Nitzsche, F.; Cui, L.; Jolkkonen, J.; Walczak, P.; Lukomska, B.; Janowski, M. Translation, but not transfection limits clinically relevant, exogenous mRNA based induction of alpha-4 integrin expression on human mesenchymal stem cells. Sci. Rep. 2017, 7, 1103.

34. Enuameh, M.S.; Kumari, S.; Zou, C.; Foulke, J.; Turner, E.; Shu, W.; Newman, R. Abstract 2012: 
The generation of a NSCLC EMT reporter cell line for metastatic lung cancer drug discovery and development. Cancer Res. 2018, 78, 2012-2012.

35. Danielsson, F.; Peterson, M.K.; Caldeira Araújo, H.; Lautenschläger, F.; Gad, A.K.B. Vimentin

36. Virtakoivu, R.; Mai, A.; Mattila, E.; De Franceschi, N.; Imanishi, S.Y.; Corthals, G.; Kaukonen,

R.; Saari, M.; Cheng, F.; Torvaldson, E.; et al. Vimentin-ERK Signaling Uncouples Slug Gene Regulatory Function. Cancer Res. 2015, 75, 2349-62.

37. Antfolk, D.; Sjöqvist, M.; Cheng, F.; Isoniemi, K.; Duran, C.L.; Rivero-Muller, A.; Antila, C.;

38. Kim, J.H.; Lee, S.-R.; Li, L.-H.; Park, H.-J.; Park, J.-H.; Lee, K.Y.; Kim, M.-K.; Shin, B.A.; Choi, S.-Y. High Cleavage Efficiency of a 2A Peptide Derived from Porcine Teschovirus-1 in Human

39. ten Dam, E.; Donnelly, M.L.L.; Gani, D.; Luke, G.; Ryan, M.D.; Mendoza, H.; Hughes, L.E. The

40. Szymczak, A.L.; Workman, C.J.; Wang, Y.; Vignali, K.M.; Dilioglou, S.; Vanin, E.F.; Vignali,

41. Liu, Z.; Chen, O.; Wall, J.B.J.; Zheng, M.; Zhou, Y.; Wang, L.; Ruth Vaseghi, H.; Qian, L.; Liu,

J. Systematic comparison of $2 \mathrm{~A}$ peptides for cloning multi-genes in a polycistronic vector. Sci. Rep. 2017, 7, 2193.

42. Rice, B.W.; Cable, M.D.; Nelson, M.B. In vivo imaging of light-emitting probes. J. Biomed. Opt.

43. Chu, J.; Haynes, R.D.; Corbel, S.Y.; Li, P.; González-González, E.; Burg, J.S.; Ataie, N.J.; Lam, A.J.; Cranfill, P.J.; Baird, M.A.; et al. Non-invasive intravital imaging of cellular differentiation with a bright red-excitable fluorescent protein. Nat. Methods 2014, 11, 572-578.

44. Kredel, S.; Nienhaus, K.; Oswald, F.; Wolff, M.; Ivanchenko, S.; Cymer, F.; Jeromin, A.; Michel, 
47. McCormick, M.B.; Kouklis, P.; Syder, A.; Fuchs, E. The roles of the rod end and the tail in vimentin IF assembly and IF network formation. J. Cell Biol. 1993, 122, 395-407.

48. Rastogi, I.; Rajanna, S.; Webb, A.; Chhabra, G.; Foster, B.; Webb, B.; Puri, N. Mechanism of cMet and EGFR tyrosine kinase inhibitor resistance through epithelial mesenchymal transition in non-small cell lung cancer. Biochem. Biophys. Res. Commun. 2016, 477, 937-944.

49. Lee, B.; Villarreal-Ponce, A.; Fallahi, M.; Ovadia, J.; Sun, P.; Yu, Q.-C.; Ito, S.; Sinha, S.; Nie, Q.; of Epidermal Progenitor Cells. Dev. Cell 2014, 29, 47-58.

50. Watanabe, K.; Villarreal-Ponce, A.; Sun, P.; Salmans, M.L.; Fallahi, M.; Andersen, B.; Dai, X.

51. Bracken, C.P.; Gregory, P.A.; Kolesnikoff, N.; Bert, A.G.; Wang, J.; Shannon, M.F.; Goodall,

52. Gregory, P.A.; Bert, A.G.; Paterson, E.L.; Barry, S.C.; Tsykin, A.; Farshid, G.; Vadas, M.A.; mesenchymal transition by targeting ZEB1 and SIP1. Nat. Cell Biol. 2008, 10, 593-601.

53. Beji, S.; Milano, G.; Scopece, A.; Cicchillitti, L.; Cencioni, C.; Picozza, M.; D’Alessandra, Y.; Pizzolato, S.; Bertolotti, M.; Spaltro, G.; et al. Doxorubicin upregulates CXCR4 via miR200c/ZEB1-dependent mechanism in human cardiac mesenchymal progenitor cells. Cell Death Dis. 2017, 8, e3020.

54. Zhou, X.; Men, X.; Zhao, R.; Han, J.; Fan, Z.; Wang, Y.; Lv, Y.; Zuo, J.; Zhao, L.; Sang, M.; et al.

55. Chen, Z.; Tang, Z.-Y.; He, Y.; Liu, L.-F.; Li, D.-J.; Chen, X. miRNA-205 Is a Candidate Tumor

56. Zhang, P.; Wang, L.; Rodriguez-Aguayo, C.; Yuan, Y.; Debeb, B.G.; Chen, D.; Sun, Y.; You, miR-200c inhibits TGF- $\beta$-induced-EMT to restore trastuzumab sensitivity by targeting ZEB1 and ZEB2 in gastric cancer. Cancer Gene Ther. 2018, 25, 68-76. Repressors ZEB1 and ZEB2. J. Biol. Chem. 2008, 283, 14910-14914. 
(c) (P) (C) 2019 by the authors. Submitted for possible open access publication under the terms and conditions of the Creative Commons Attribution (CC BY) license (http://creativecommons.org/licenses/by/4.0/). 